\title{
A relaxation theorem for a differential inclusion with "maxima"
}

\author{
Aurelian Cernea
}

\begin{abstract}
We consider a Cauchy problem associated to a nonconvex differential inclusion with "maxima" and we prove a Filippov type existence result. This result allows to obtain a relaxation theorem for the problem considered.
\end{abstract}

\section{Indroduction}

Differential equations with maximum have proved to be strong tools in the modelling of many physical problems: systems with automatic regulation, problems in control theory that correspond to the maximal deviation of the regulated quantity etc.. As a consequence there was an intensive development of the theory of differential equations with "maxima" $[2,5,6,8-14]$ etc..

A classical example is the one of an electric generator ([2]). In this case the mechanism becomes active when the maximum voltage variation is reached in an interval of time. The equation describing the action of the regulator has the form

$$
x^{\prime}(t)=a x(t)+b \max _{s \in[t-h, t]} x(s)+f(t),
$$

where $a, b$ are constants given by the system, $x($.$) is the voltage and f($.$) is a$ perturbation given by the change of voltage.

In this paper we study the following problem

$$
x^{\prime}(t) \in F\left(t, x(t), \max _{s \in[0, t]} x(s)\right) \quad \text { a.e. }([0,1]), \quad x(0)=x_{0}
$$

Key Words: Set-valued map, differential inclusion, relaxation.

2010 Mathematics Subject Classification: Primary 34A60; Secondary 34K10.

Received: 20.02 .2016

Accepted: 25.04.2016 
where $F:[0,1] \times \mathbb{R} \times \mathbb{R} \rightarrow \mathcal{P}(\mathbb{R})$ is a set-valued map and $x_{0} \in \mathbb{R}$. Several existing results for problem (1) obtained with fixed point approaches may be found in our previous paper [3].

The aim of this note is to obtain a relaxation theorem for the problem considered. Namely, we prove that the solution set of the problem (1) is dense in the set of the relaxed solutions; i.e. the set of solutions of the differential inclusion whose right hand side is the convex hull of the original set-valued map. In order to prove this result we show, first, that Filippov's ideas ([4]) can be suitably adapted in order to obtain the existence of solutions of problem (1). We recall that for a differential inclusion defined by a lipschitzian setvalued map with nonconvex values Filippov's theorem ([4]) consists in proving the existence of a solution starting from a given "quasi" solution. Moreover, the result provides an estimate between the starting "quasi" solution and the solution of the differential inclusion.

The paper is organized as follows: in Section 2 we briefly recall some preliminary results that we will use in the sequel and in Section 3 we prove the main results of the paper.

\section{Preliminaries}

In this section we sum up some basic facts that we are going to use later.

Let $(X, d)$ be a metric space. The Pompeiu-Hausdorff distance of the closed subsets $A, B \subset X$ is defined by $d_{H}(A, B)=\max \left\{d^{*}(A, B), d^{*}(B, A)\right\}$, $d^{*}(A, B)=\sup \{d(a, B) ; a \in A\}$, where $d(x, B)=\inf \{d(x, y) ; y \in B\}$. Let $I:=[0,1]$ and denote by $\mathcal{L}(I)$ the $\sigma$-algebra of all Lebesgue measurable subsets of $I$. Denote by $\mathcal{P}(\mathbb{R})$ the family of all nonempty subsets of $\mathbb{R}$ and by $\mathcal{B}(\mathbb{R})$ the family of all Borel subsets of $\mathbb{R}$. For any subset $A \subset \mathbb{R}$ we denote by $\operatorname{cl} A$ the closure of $A$ and by $\overline{\mathrm{co}}(A)$ the closed convex hull of $A$.

As usual, we denote by $C(I, \mathbb{R})$ the Banach space of all continuous functions $x():. I \rightarrow \mathbb{R}$ endowed with the norm $|x|_{C}=\sup _{t \in I}|x(t)|$ and by $L^{1}(I, \mathbb{R})$ the Banach space of all integrable functions $x():. I \rightarrow \mathbb{R}$ endowed with the norm $|x|_{1}=\int_{0}^{T}|x(t)| \mathrm{d} t$. The Banach space of all absolutely continuous functions $x():. I \rightarrow \mathbb{R}$ will be denoted by $A C(I, \mathbb{R})$. We recall that for a set-valued map $U: I \rightarrow \mathcal{P}(\mathbb{R})$ the Aumann integral of $\mathrm{U}$, denoted by $\int_{I} U(t) \mathrm{d} t$, is the set

$$
\int_{I} U(t) \mathrm{d} t=\left\{\int_{I} u(t) \mathrm{d} t ; u(.) \in L^{1}(I, \mathbb{R}), u(t) \in U(t) \text { a.e. }(I)\right\}
$$

We recall two results that we are going to use in the next section. The first one is a selection result (e.g., [1]) which is a version of the celebrated Kuratowski and Ryll-Nardzewski selection theorem. The proof of the second one may be found in [7]. 
Lemma 1. Consider $X$ a separable Banach space, $B$ is the closed unit ball in $X, H: I \rightarrow \mathcal{P}(X)$ is a set-valued map with nonempty closed values and $g: I \rightarrow X, L: I \rightarrow \mathbb{R}_{+}$are measurable functions. If

$$
H(t) \cap(g(t)+L(t) B) \neq \emptyset \quad \text { a.e. }(I),
$$

then the set-valued map $t \rightarrow H(t) \cap(g(t)+L(t) B)$ has a measurable selection.

Lemma 2. Let $U: I \rightarrow \mathcal{P}(\mathbb{R})$ be a measurable set-valued map with closed nonempty images and having at least one integrable selection. Then

$$
\operatorname{cl}\left(\int_{0}^{T} \overline{\operatorname{co}} U(t) \mathrm{d} t\right)=\operatorname{cl}\left(\int_{0}^{T} U(t) \mathrm{d} t\right) .
$$

Let $I():. \mathbb{R} \rightarrow \mathcal{P}(\mathbb{R})$ a set-valued map with compact convex values defined by $I(t)=[a(t), b(t)]$, where $a(),. b():. \mathbb{R} \rightarrow \mathbb{R}$ are continuous functions with $a(t) \leq b(t) \forall t \in \mathbb{R}$. For $x():. \mathbb{R} \rightarrow \mathbb{R}$ continuous we define $\left(\max _{I}\right)(t)=$ $\max _{s \in I(t)} x(s)$. Therefore, $\max _{I}: C(\mathbb{R}, \mathbb{R}) \rightarrow C(\mathbb{R}, \mathbb{R})$ is an operator whose properties are summarized in the next lemma proved in [12].

Lemma 3. If $x(),. y(.) \in C(\mathbb{R}, \mathbb{R})$, then one has

i) $\left|\max _{s \in I(t)} x(s)-\max _{s \in I(t)} y(s)\right| \leq \max _{s \in I(t)}|x(s)-y(s)| \forall t \in \mathbb{R}$. $\forall t \in \mathbb{R}$.

ii) $\max _{t \in K}\left|\max _{s \in I(t)} x(s)-\max _{s \in I(t)} y(s)\right| \leq \max _{s \in \cup_{t \in K} I(t)}|x(s)-y(s)|$

\section{The main results}

In what follows we assume the following hypotheses.

Hypothesis. i) $F(., .,):. I \times \mathbb{R} \times \mathbb{R} \rightarrow \mathcal{P}(\mathbb{R})$ has nonempty closed values and is $\mathcal{L}(I) \otimes \mathcal{B}(\mathbb{R} \times \mathbb{R})$ measurable.

ii) There exist $l_{1}(),. l_{2}(.) \in L^{1}\left(I, \mathbb{R}_{+}\right)$such that, for almost all $t \in I$,

$\mathrm{d}_{H}\left(F\left(t, x_{1}, y_{1}\right), F\left(t, x_{2}, y_{2}\right)\right) \leq l_{1}(t)\left|x_{1}-x_{2}\right|+l_{2}(t)\left|y_{1}-y_{2}\right| \forall x_{1}, x_{2}, y_{1}, y_{2} \in \mathbb{R}$.

Theorem 1. Assume Hypothesis satisfied and $\left|l_{1}\right|_{1}+\left|l_{2}\right|_{1}<1$. Let $y(.) \in$ $A C(I, \mathbb{R})$ be such that there exists $p(.) \in L^{1}\left(I, \mathbb{R}_{+}\right)$verifying $d\left(y^{\prime}(t), F(t\right.$, $\left.\left.y(t), \max _{s \in[0, t]} y(s)\right)\right) \leq p(t)$ a.e. $(I)$.

Then there exists $x($.$) a solution of problem (1) satisfying for all t \in I$

$$
|x-y|_{C} \leq \frac{1}{1-\left(\left|l_{1}\right|_{1}+\left|l_{2}\right|_{1}\right)}\left(\left|x_{0}-y(0)\right|+|p|_{1}\right) .
$$


Proof. We set $x_{0}()=.y(),. f_{0}()=.y^{\prime}($.$) .$

The set-valued map $t \rightarrow F\left(t, y(t), \max _{s \in[0, t]} y(s)\right)$ is measurable with closed values and

$$
F\left(t, y(t), \max _{s \in[0, t]} y(s)\right) \cap\left\{y^{\prime}(t)+p(t)[-1,1]\right\} \neq \emptyset \quad \text { a.e. }(I) .
$$

It follows from Lemma 1 that there exists a measurable function $f_{1}($.$) such$ that $f_{1}(t) \in F\left(t, x_{0}(t), \max _{s \in[0, t]} x_{0}(s)\right)$ a.e. ( $\left.I\right)$ and, for almost all $t \in I$, $\left|f_{1}(t)-y^{\prime}(t)\right| \leq p(t)$. Define $x_{1}(t)=x_{0}+\int_{0}^{t} f_{1}(s) d s$ and one has

$$
\left|x_{1}(t)-y(t)\right| \leq\left|x_{0}-y(0)\right|+\int_{0}^{t} p(s) d s \leq\left|x_{0}-y(0)\right|+|p|_{1} .
$$

Thus $\left|x_{1}-y\right|_{C} \leq\left|x_{0}-y(0)\right|+|p|_{1}$.

The set-valued map $t \rightarrow F\left(t, x_{1}(t), \max _{s \in[0, t]} x_{1}(s)\right)$ is measurable. Moreover, the map $t \rightarrow l_{1}(t)\left|x_{1}(t)-x_{0}(t)\right|+l_{2}(t)\left|\max _{s \in[0, t]} x_{1}(s)-\max _{s \in[0, t]} x_{0}(s)\right|$ is measurable. By the lipschitzianity of $F(t, .,$.$) we have that for almost all$ $t \in I$

$$
\begin{gathered}
d\left(f_{1}(t), F\left(t, x_{1}(t), \max _{s \in[0, t]} x_{1}(s)\right)\right) \leq d_{H}\left(F\left(t, x_{0}(t), \max _{s \in[0, t]} x_{0}(s)\right),\right. \\
\left.F\left(t, x_{1}(t), \max _{s \in[0, t]} x_{1}(s)\right)\right) \leq l_{1}(t)\left|x_{1}(t)-x_{0}(t)\right|+l_{2}(t)\left|\max _{s \in[0, t]} x_{0}(s)-\max _{s \in[0, t]} x_{1}(s)\right| .
\end{gathered}
$$

Therefore,

$$
\begin{aligned}
& \left.F\left(t, x_{1}(t), \max _{s \in[0, t]} x_{1}(s)\right)\right) \cap\left\{f_{1}(t)+\left(l_{1}(t)\left|x_{1}(t)-x_{0}(t)\right|+\right.\right. \\
& \left.\left.l_{2}(t)\left|\max _{s \in[0, t]} x_{1}(s)-\max _{s \in[0, t]} x_{0}(s)\right|\right)[-1,1]\right\} \neq \emptyset .
\end{aligned}
$$

From Lemma 1 we deduce the existence of a measurable function $f_{2}($.$) such$ that $f_{2}(t) \in F\left(t, x_{1}(t), \max _{s \in[0, t]} x_{1}(s)\right)$ a.e. $(I)$ and for almost all $t \in I$

$$
\begin{aligned}
& \left|f_{1}(t)-f_{2}(t)\right| \leq d\left(f_{1}(t), F\left(t, x_{1}(t), \max _{s \in[0, t]} x_{1}(s)\right)\right) \leq d_{H}\left(F\left(t, x_{0}(t), \max _{s \in[0, t]} x_{0}(s)\right),\right. \\
& \left.F\left(t, x_{1}(t), \max _{s \in[0, t]} x_{1}(s)\right)\right) \leq l_{1}(t)\left|x_{1}(t)-x_{0}(t)\right|+l_{2}(t)\left|\max _{s \in[0, t]} x_{0}(s)-\max _{s \in[0, t]} x_{1}(s)\right| .
\end{aligned}
$$

Define $x_{2}(t)=x_{0}+\int_{0}^{t} f_{2}(s) d s$ and one has

$$
\begin{gathered}
\left|x_{1}(t)-x_{2}(t)\right| \leq \int_{0}^{t}\left|f_{1}(s)-f_{2}(s)\right| d s \leq \int_{0}^{t}\left[l_{1}(s)\left|x_{0}(s)-x_{1}(s)\right|+\right. \\
\left.l_{2}(s)\left|\max _{\sigma \in[0, s]} x_{0}(\sigma)-\max _{\sigma \in[0, s]} x_{1}(\sigma)\right|\right] d s \leq\left(\left|l_{1}\right|_{1}+\left|l_{2}\right|_{1}\right)\left|x_{1}-x_{0}\right|_{C}
\end{gathered}
$$




$$
\leq\left(\left|l_{1}\right|_{1}+\left|l_{2}\right|_{1}\right)\left(\left|x_{0}-y(0)\right|+|p|_{1}\right) .
$$
fying

Assume that for some $n \geq 1$ we have constructed $\left(x_{i}(.)\right)_{i=1}^{n}$ with $x_{n}$ satis-

$$
\left|x_{n}-x_{n-1}\right|_{C} \leq\left(\left|l_{1}\right|_{1}+\left|l_{2}\right|_{1}\right)^{n-1}\left(\left|x_{0}-y(0)\right|+|p|_{1}\right) .
$$

The set-valued map $t \rightarrow F\left(t, x_{n}(t), \max _{s \in[0, t]} x_{n}(s)\right)$ is measurable. At the same time, the map $t \rightarrow l_{1}(t)\left|x_{n}(t)-x_{n-1}(t)\right|+l_{2}(t) \mid \max _{s \in[0, t]} x_{n}(s)-$ $\max _{s \in[0, t]} x_{n-1}(s) \mid$ is measurable. As before, by the lipschitzianity of $F(t, .,$. we have that for almost all $t \in I$

$$
\begin{aligned}
& \left.F\left(t, x_{n}(t), \max _{s \in[0, t]} x_{n}(s)\right)\right) \cap\left\{f_{n}(t)+\left(l_{1}(t)\left|x_{n}(t)-x_{n-1}(t)\right|+\right.\right. \\
& \left.\left.l_{2}(t)\left|\max _{s \in[0, t]} x_{n}(s)-\max _{s \in[0, t]} x_{n-1}(s)\right|\right)[-1,1]\right\} \neq \emptyset .
\end{aligned}
$$

Using again Lemma 1 we deduce the existence of a measurable function $f_{n+1}($.$) such that f_{n+1}(t) \in F\left(t, x_{n}(t), \max _{s \in[0, t]} x_{n}(s)\right)$ a.e. $(I)$ and for almost all $t \in I$

$$
\begin{gathered}
\left|f_{n+1}(t)-f_{n}(t)\right| \leq d\left(f_{n+1}(t), F\left(t, x_{n-1}(t), \max _{s \in[0, t]} x_{n-1}(s)\right)\right) \leq \\
d_{H}\left(F\left(t, x_{n}(t), \max _{s \in[0, t]} x_{n}(s)\right), F\left(t, x_{n-1}(t), \max _{s \in[0, t]} x_{n-1}(s)\right)\right) \leq \\
l_{1}(t)\left|x_{n}(t)-x_{n-1}(t)\right|+l_{2}(t)\left|\max _{s \in[0, t]} x_{n}(s)-\max _{s \in[0, t]} x_{n-1}(s)\right| .
\end{gathered}
$$

Define

$$
x_{n+1}(t)=x_{0}+\int_{0}^{t} f_{n+1}(s) d s .
$$

We have

$$
\begin{gathered}
\left|x_{n+1}(t)-x_{n}(t)\right| \leq \int_{0}^{t}\left|f_{n+1}(s)-f_{n}(s)\right| d s \leq \\
\int_{0}^{t}\left[l_{1}(s)\left|x_{n}(s)-x_{n-1}(s)\right|+l_{2}(s)\left|\max _{\sigma \in[0, s]} x_{n}(\sigma)-\max _{\sigma \in[0, s]} x_{n-1}(\sigma)\right|\right] d s \\
\leq\left(\left|l_{1}\right|_{1}+\left|l_{2}\right|_{1}\right)\left|x_{n}-x_{n-1}\right|_{C} \leq\left(\left|l_{1}\right|_{1}+\left|l_{2}\right|_{1}\right)^{n}\left(\left|x_{0}-y(0)\right|+|p|_{1}\right) .
\end{gathered}
$$

Therefore $\left(x_{n}(.)\right)_{n \geq 0}$ is a Cauchy sequence in the Banach space $C(I, \mathbb{R})$, so it converges to $x(.) \in C(I, \mathbb{R})$. Since, for almost all $t \in I$, we have

$$
\begin{gathered}
\left|f_{n+1}(t)-f_{n}(t)\right| \leq l_{1}(t)\left|x_{n}(t)-x_{n-1}(t)\right|+l_{2}(t)\left|\max _{s \in[0, t]} x_{n}(t)-\max _{s \in[0, t]} x_{n-1}(t)\right| \\
\leq\left[l_{1}(t)+l_{2}(t)\right]\left|x_{n}-x_{n-1}\right|_{C},
\end{gathered}
$$

$\left\{f_{n}().\right\}$ is a Cauchy sequence in the Banach space $L^{1}(I, \mathbb{R})$ and thus it converges to $f(.) \in L^{1}(I, \mathbb{R})$. 
We note that one may write

$$
\begin{aligned}
\left|\int_{0}^{t} f_{n}(s) d s-\int_{0}^{t} f(s) d s\right| & \leq \int_{0}^{t}\left|f_{n}(s)-f(s)\right| d s \leq \int_{0}^{t}\left[l_{1}(s)+l_{2}(s)\right]\left|x_{n+1}-x\right|_{C} d s \\
& \leq\left(\left|l_{1}\right|_{1}+\left|l_{2}\right|_{1}\right) \cdot\left|x_{n+1}-x\right|_{C} .
\end{aligned}
$$

Therefore, one may pass to the limit in (3) and we get $x(t)=x_{0}+\int_{0}^{t} f(s) d s$. Moreover, since the values of $F(., . .$.$) are closed and f_{n+1}(t) \in F\left(t, x_{n}(t)\right.$,

$\left.\max _{s \in[0, t]} x_{n}(t)\right)$ passing to the limit we obtain $f(t) \in F\left(t, x(t), \max _{s \in[0, t]} x(t)\right)$ a.e. $(I)$.

It remains to prove the estimate (2). One has

$$
\begin{gathered}
\left|x_{n}-x_{0}\right|_{C} \leq\left|x_{n}-x_{n-1}\right|_{C}+\ldots+\left|x_{2}-x_{1}\right|_{C}+\left|x_{1}-x_{0}\right|_{C} \leq \\
\left(\left|l_{1}\right|_{1}+\left|l_{2}\right|_{1}\right)^{n-1}\left(\left|x_{0}-y(0)\right|+|p|_{1}\right)+\ldots+\left(\left|l_{1}\right|_{1}+\left|l_{2}\right|_{1}\right)\left(\left|x_{0}-y(0)\right|+|p|_{1}\right)+ \\
\left(\left|x_{0}-y(0)\right|+|p|_{1}\right) \leq \frac{1}{1-\left(\left|l_{1}\right|_{1}+\left|l_{2}\right|_{1}\right)}\left(\left|x_{0}-y(0)\right|+|p|_{1}\right) .
\end{gathered}
$$

Passage to the limit in the last inequality completes the proof.

Remark 1. A similar result to the one in Theorem 1 may be found in [3], namely Theorem 3.1. The approach in [3], apart from the requirement that the values of $F(.,$.$) are compact, does not provides a priori bounds for solutions$ as in (3.1).

As we already pointed out, Theorem 1 allows to obtain a relaxation theorem for problem (1). In what follows, we are concerned also with the convexified (relaxed) problem

$$
x^{\prime}(t) \in \overline{\mathrm{co}} F\left(t, x(t), \max _{s \in[0, t]} x(s)\right), \quad x(0)=x_{0} .
$$

Note that if $F(., .,$.$) satisfies Hypothesis, then so does the set-valued map$ $(t, x, y) \rightarrow \overline{\mathrm{co}} F(t, x, y)$ (e.g., [1]).

Theorem 2. We assume that Hypothesis is satisfied and $\left|l_{1}\right|_{1}+\left|l_{2}\right|_{1}<1$. Let $\bar{x}():. I \rightarrow \mathbb{R}$ be a solution to the relaxed inclusion (4) such that the set-valued map $t \rightarrow F\left(t, \bar{x}(t), \max _{s \in[0, t]} \bar{x}(s)\right)$ has at least one integrable selection.

Then for every $\varepsilon>0$ there exists $x($.$) a solution of problem (1) such that$

$$
|x-\bar{x}|_{C}<\varepsilon \text {. }
$$

Proof. Since $\bar{x}($.$) is a solution of the relaxed inclusion (4), there exists \bar{f}(.) \in$ $L^{1}(I, \mathbb{R}), \bar{f}(t) \in \overline{\mathrm{co}} F\left(t, \bar{x}(t), \max _{s \in[0, t]} \bar{x}(s)\right)$ a.e. $(I)$ such that $\bar{x}(t)=x_{0}+$ $\int_{0}^{t} \bar{f}(s) d s$. 
From Lemma 2, for $\delta>0$, there exists $\tilde{f}(t) \in F\left(t, \bar{x}(t), \max _{s \in[0, t]} \bar{x}(s)\right)$ a.e. (I) such that

$$
\sup _{t \in I}\left|\int_{0}^{t}(\tilde{f}(s)-\bar{f}(s)) d s\right| \leq \delta
$$

Define $\tilde{x}(t)=x_{0}+\int_{0}^{t} \tilde{f}(s) d s$. Therefore, $|\tilde{x}-\bar{x}|_{C} \leq \delta$.

We apply Theorem 1 for the "quasi" solution $\tilde{x}($.$) of (1). One has$

$$
\begin{gathered}
p(t)=d\left(\tilde{f}(t), F\left(t, \tilde{x}(t), \max _{s \in[0, t]} \tilde{x}(s)\right)\right) \leq d_{H}\left(F\left(t, \bar{x}(t), \max _{s \in[0, t]} \bar{x}(s)\right),\right. \\
\left.F\left(t, \tilde{x}(t), \max _{s \in[0, t]} \tilde{x}(s)\right)\right) \leq l_{1}(t)|\bar{x}(t)-\tilde{x}(t)|+l_{2}(t)\left|\max _{s \in[0, t]} \bar{x}(s)-\max _{s \in[0, t]} \tilde{x}(s)\right| \\
\leq l_{1}(t)|\tilde{x}-\bar{x}|_{C}+l_{2}(t)|\tilde{x}-\bar{x}|_{C} \leq\left(l_{1}(t)+l_{2}(t)\right) \delta,
\end{gathered}
$$

which shows that $p(.) \in L^{1}(I, \mathbb{R})$.

From Theorem 1 there exists $x($.$) a solution of (1) such that$

$$
|x-\tilde{x}|_{C} \leq \frac{1}{1-\left(\left|l_{1}\right|_{1}+\left|l_{2}\right|_{1}\right)}|p|_{1} \leq \frac{\left|l_{1}\right|_{1}+\left|l_{2}\right|_{1}}{1-\left(\left|l_{1}\right|_{1}+\left|l_{2}\right|_{1}\right)} \delta .
$$

It remains to take $\delta=\left[1-\left(\left|l_{1}\right|_{1}+\left|l_{2}\right|_{1}\right)\right] \varepsilon$ and to deduce that $|x-\bar{x}|_{C} \leq$ $|x-\tilde{x}|_{C}+|\tilde{x}-\bar{x}|_{C} \leq \varepsilon$.

\section{References}

[1] Aubin, J.P., Frankowska, H., Set-valued Analysis, Birkhauser, Basel, 1990.

[2] Bainov, D.D., Hristova, S., Differential equations with maxima, Chapman and Hall/CRC, Boca Raton, 2011.

[3] Cernea, A., On the existence of solutions for differential inclusions with "maxima", Libertas Mathematica, New Series 35 (2015), 89-98.

[4] Filippov, A.F., Classical solutions of differential equations with multivalued right hand side, SIAM J. Control 5 (1967), 609-621.

[5] Georgiev, L., Angelov, V.G., On the existence and uniqueness of solutions for maximum equations, Glasnik Mat. 37 (2002), 275-281.

[6] Gonzalez, P., Pinto, M., Convergent solutions of certain nonlinear differential equations with maxima, Math. Comput. Modelling 45 (2007), $1-10$. 
[7] Hiai, F., Umegaki, H., Integrals, Conditional Expectations and Martingales of Multivalued Functions, J. Multivariate Anal. 7 (1977), 149-182.

[8] Ivanov, A., Liz, E., Trofimchuk, S., Halanay inequality, Yorke 3/2 stability criteria and differential equations with maxima, Tohoku Math. J. 54 (2002), 277-295.

[9] Malgorzata, M., Zhang, G., On unstable neutral difference equations with "maxima", Math. Slovaca 56 (2006), 451-463.

[10] Otrocol, D., Systems of functional differential equations with maxima, of mixed type, Electronic J. Qual. Theory Differ. Equations 2014 (2014), no. $5,1-9$.

[11] Otrocol, D., Rus, I.A., Functional-differential equations with "maxima" via weakly Picard operator theory, Bull. Math. Soc. Sci. Math. Roumanie 51(99) (2008), 253-261.

[12] Otrocol, D., Rus, I.A., Functional-differential equations with "maxima", of mixed type, Fixed Point Theory 9 (2008), 207-220.

[13] Popov, E.P., Automatic regulation and control, Nauka, Moskow, 1966 (in Russian).

[14] Stepanov, E., On solvability of some boundary value problems for differential equations with "maxima", Topol. Meth. Nonlin. Anal. 8 (1996), 315-326.

Aurelian CERNEA,

Faculty of Mathematics and Computer Science,

University of Bucharest,

Academiei 14, 010014 Bucharest, Romania.

Academy of Romanian Scientists,

Splaiul Independenţei 54, 050094 Bucharest, Romania.

Email: acernea@fmi.unibuc.ro 